\title{
Arbeitshygiene in schweizerischen Kalendern des 19. Jahrhunderts
}

\author{
Von Barbara C. Hansch-Mock
}

Über Arbeitshygiene läßt sich aus den schweizerischen Kalendern des 19. Jahrhunderts manches entnehmen. Für den Volkskundler und Medizinhistoriker bilden sie wertvolle und ergiebige Quellen. Da diese Kalender sich beim Volk großer Beliebtheit erfreuten (denn Massenmedien wie Radio und Fernsehen standen ja noch nicht zur Verfügung), konnten die Redaktoren durch sie allgemeine Kenntnisse verbreiten.

Daß die Gesundheit als einer der wichtigsten Faktoren des menschlichen Lebens gilt, läßt sich an Hand von vielen hygienischen Regeln durch die gesamte Kalenderliteratur verfolgen. Allgemein herrschte in den Kalendern die Meinung, daß der Mensch jede Krankheit selbst verschulde. So wurde als Teil der Volksgesundheitspflege in der zweiten Hälfte des 19. Jahrhunderts die Arbeitshygiene in Industrie und Gewerbe in zunehmendem Maße in den Kalendern behandelt. Hier wurde auf die mit der Berufsausübung in Zusammenhang stehenden gesundheitlichen Schädigungen hingewiesen, und man versuchte sie durch prophylaktische Gegenmaßnahmen zu bekämpfen. Körperliche Leistungsfähigkeit und Widerstandskraft der erwerbstätigen Bevölkerung sollten soweit wie möglich erhalten werden.

Die Entwicklung des Arbeiterschutzes verlief bekanntlich sehr langsam. Da es im Altertum und im Mittelalter keinen Arbeiterschutz gab und den Arbeitsgefahren keinerlei Aufmerksamkeit geschenkt wurde, finden wir für jene Zeit nur vereinzelte Angaben über berufliche Schädigungen ${ }^{1}$. Erst das 15. Jahrhundert zeigte vermehrtes Interesse für Berufskrankheiten und ihre Prophylaxe ${ }^{2}$. Mit dem Beginn der Industrie entstand eine wissenschaftliche Literatur über Berufskrankheiten. Als Begründer der Arbeitsmedizin gilt der 1633 in Capri geborene Medizinprofessor Bernardino Ramazzini, der als erster die Wichtigkeit seiner Wissenschaft bei der Beurteilung der verschiedenen Berufskrankheiten erkannte. Dies geschah in seinem Hauptwerk «De morbis artificum diatriba» $(1700)^{3}$. Seine Studien bilden im 18. und 19. Jahrhundert den Ausgangspunkt weiterer Untersuchungen und Veröffentlichungen. Deutsche Gelehrte des 18. Jahrhunderts beschäftigten sich in erster Linie mit den Leiden der Geistesarbeiter, darunter Mediziner, Theolo- 
gen, Mönche und Studenten. Die Krankheiten der Handwerker wurden weitgehend außer acht gelassen ${ }^{4}$.

Ramazzinis Ausführungen hatten auf die Studien verschiedener Schweizer Ärzte Einfluß. Besonders Fridolin Schuler aus Mollis (1832-1903) deutete auf die schädigenden Wirkungen einiger Arbeitsprozesse in den Fabriken hin ${ }^{5}$. Er führte in der eidgenössischen Fabrikinspektion seinen unermüdlichen Kampf gegen die Entstehung der Berufskrankheiten ${ }^{6}$. Auch Jakob Laurenz Sonderegger beschäftigte sich mit der Gefährdung durch bestimmte Berufe und erkannte, daß der Beruf die soziale Stellung, die Gesundheit und die Lebensdauer bedingt ${ }^{7}$. Nebst der Erkenntnis, daß verschiedene Tätigkeiten bestimmte Krankheiten verursachen können, hielten die Kalender daran fest, daß «eine ungesunde Lebensweise der Grund zu mancherlei Krankheitsanlagen und wirklichen Krankheiten» ist (Zuger Kalender, Zug 1869). Da diese Tatsache einen wesentlichen Einfluß auf das Wohl der größten Bevölkerungsklasse ausübt, hielten es die Kalenderschreiber für ihre Pflicht, das Problem einer «einläßlichen Besprechung» zu würdigen. An Hand der Kalender läßt sich die nachteilige Wirkung verschiedener Berufe auf den Arbeiter ermitteln.

Über Schneider heißt es, daß sie in der Regel magere Gestalten sind, was sicherlich daran liegt, daß es zur Ausübung dieses Berufes keinen sonderlichen Kraftaufwand erforderte. Im Zuger Kalender von 1869 steht: «Schneider sind meist schwächliche Menschen und selten wählt ein gesunder, kräftiger Bursche das Schneiderhandwerk.» Die sitzende Lebensweise, die gebückte Stellung führte häufig zu Krankheiten der Lunge und der Unterleibsorgane. Fridolin Schulers Untersuchungen bestätigen, daß außer bei den Fabrikarbeitern auch unter den Schneidern und anderen Professionen «eine beträchtliche Zahl Schwächlinge von Anbeginn» zu finden ist, «bei denen noch eine sehr mangelhafte Körperbeschaffenheit die Ausübung des Berufes gestattet». ${ }^{8} \mathrm{Da}$ für den Schneider das Einatmen von Kleiderstaub die Lunge gefährdet, ist einleuchtend ${ }^{9}$. Neben Lungenerkrankungen sah man in den Kalendern das Sitzen als besonders gefährdendes Moment an. Wie Sonderegger anführt, wird nach Forschungen in Frankfurt nur die Hälfte der Schneider älter als 42 Jahre, und 40 bis 42 von Hundert fallen der Lungenschwindsucht zum Opfer ${ }^{10}$. Den Angaben von Braune zufolge hatten die Schneidergesellen damals in Leipzig sogar ihr eigenes Krankenhaus ${ }^{11}$. Zur Abhilfe dieser Berufskrankheiten empfiehlt der «Zuger Kalender» von 1869 «häufige Lüftung der Arbeitsräume, Wechseln der Wäsche, Hautpflege, und öftere Bewegung und freie Luft». 
Den Schuhmachern ging es in gesundheitlicher Hinsicht nicht besser als den Schneidern. Im «St.Galler Kalender» von 1865 heißt es: «Sie sind zumeist mit unverhältnismäßigen Gliedern des Unterleibs begabt, was von verstickter Luft bei stetem Sitzen auf konvexem Stuhl herkommen mag. Diese dringt allmälig auf das Innere derselben, macht sie hipochondrisch und konfus und endet nicht selten mit dem Tod.» Infolge von verstickter Luft starben in der Tat von 100 Schustern 42 an Schwindsucht und 10 bis 12 an Herzkrankheiten ${ }^{12}$. Der «Zuger Kalender» von 1869 bringt folgende Angaben über die Besonderheiten der Schuhmacher: «Schuster sind meist kräftige, muskulöse Menschen; ihre sitzende Beschäftigungsweise, die eine große Zusammenpressung der Unterleibsorgane bedingt, disponiert sie zu Unterleibskrankheiten, zu aus dieser entspringender Hypochondrie und Melancholie, woraus auch leicht zu erklären, daß Schuster es so häufig waren, die sich der religiösen Schwärmerei hingaben.» ${ }^{13}$

Über Metzger heißt es, daß sie «blutgierige Menschen» seien, die jedoch «nicht selten außerordentlich zarte Gefühle gegen Mitmenschen und besonders gegen das weibliche Geschlecht» haben (St.Galler Kalender 1865). Obwohl die Vertreter dieser Berufsgattung in der Regel «kräftig und gesund» seien, haben auch sie einige Leiden zu bekämpfen: «Vollblütigkeit mit all ihren Folgen, Fettsucht sowie die Wurmkrankheit» (Zuger Kalender 1869), was zum Teil durch ihre Unmäßigkeit im Essen gefördert wird ${ }^{14}$.

Maurer und Steinhauer haben an den Wirkungen des Staubes zu leiden. Bei ihnen kommt eine «besondere Engbrüstigkeit vor, die daher die Steinbrecherkrankheit» ${ }^{15}$ genannt wird (Zuger Kalender 1869). In Bayern stehen die Steinhauer an erster Stelle in der Liste der Berufe, deren Krankheitsfälle auf Staubeinatmung zurückgeführt werden können ${ }^{16}$. Der «Luzerner Hauskalender» von 1893 und 1895 empfiehlt ein Rezept, um Augenverletzungen durch Kalk zu vermeiden: «Arbeiter, die oft auf Bauten zu thun haben, wissen wie gefährlich es ist, wenn durch einen Zufall Kalk ins Auge gelangt. Ein einfaches Mittel hiergegen ist kaltes Zuckerwasser, mit dem das betreffende Auge ausgewaschen wird.»

Müller und Bäcker leiden oft an Schwindsucht und Wassersucht, und wegen ihrer feuchten Wohnungen sind sie zu Wechselfiebern disponiert (Zuger Kalender 1869, St. Galler Kalender 1865). Sonderegger führt den «raschen Temperaturwechsel», dem die Bäcker ausgesetzt sind, neben «Staub [Mehlstaub], Anstrengung und Verkühlung» als besondere Übel an. Daher erkranken sie oft an Brustkatarrh und «bei unzuverlässiger Constitution» an Lungenschwindsucht ${ }^{17}$. Von 100 Bäckern sterben 23 an Lungen- 
schwindsucht ${ }^{18}$. Da der Bäcker bei Nacht arbeitet, bekommt er tagsüber wenig Schlaf und wird häufig nervös, so daß 18-19\% aller Erkrankungen bei Bäckern nervöse Krankheiten mit vorwaltender Schwäche sind.

Den Seifensiedern, Bleichern und Wäschern werden Anlagen zu Augenkrankheiten und wegen der scharfen Dämpfe und Laugen Krankheiten der Atmungswege bescheinigt. Sonderegger schreibt die häufige Erkrankung der Wäscher und Färber der Nässe und Feuchtigkeit zu, der sie immer

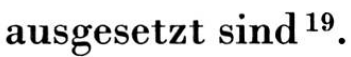

Der Beruf der Weber galt als einer der verderblichsten für die Gesundheit. Hier wird in erster Linie die Körperstellung genannt, «nämlich das Anliegen der Magengegend an den Webstuhl» (Zuger Kalender 1869). Nachteile dieser sitzenden Haltung sind «Blutstockungen, erhöhte Blutsammlung im Unterleib, was wiederum Störungen der Verdauung bewirkt». ${ }^{20}$ Als weitere krankheitsverursachende Elemente beim Gewerbe der Weber werden «Staub, stinkende Dämpfe, die sich aus den Tüchern und ranzigem Maschinenöl entwickeln», genannt, ferner die «feuchte, dunkle, meist unterirdische Wohnung und namentlich der unmäßige Branntweingenuß ... Demzufolge sind die Weber meist schwächliche, bleiche Individuen mit viel Empfänglichkeit zu Krankheiten, vorzugsweise Skrophulosis, chronische Leiden der Luftwege, Unterleibskrankheiten, Wassersucht, Wechselfieber u.a.m.» Wie den Fabrikinspektionsberichten der Jahre 1867 bis 1870 zu entnehmen ist, arbeiteten während dieser Zeit im Kanton Glarus 9500 Personen in der Baumwollindustrie, davon 3600 in Spinnereien und Webereien ${ }^{21}$. Daß selbst noch in neuerer Zeit 20,2 \% aller Fabrikarbeiter Krankheiten der Atmungsorgane erliegen, beweist eine 1955 für Hamburg durchgeführte Studie ${ }^{22}$. In der Schweiz wurde versucht, die Ansteckungsgefahr der Tuberkulose in den Fabriken zu bekämpfen, indem man Spuckverbote erließ; wie ein Aufsichtsbeamter bemerkte, war es aber besonders schwer, «die schlechte Gewohnheit des Ausspuckens auf den Boden zu beseitigen». ${ }^{23}$ Als Maßnahmen gegen die genannten Übel erteilt der Zuger Kalender von 1869 folgende Ratschläge: «Häufige Lüftung der Arbeitsräume, Reinlichkeit und häufiges Wechseln der Wäsche, Hautpflege und viel Bewegung in freier Luft, Vermeidung blähender Speisen und geistiger Getränke.» Oder: «Geistige Beschäftigung in freien Stunden, deren Weber mehr bedürfen als die meisten anderen Professionisten, ist hier von Wichtigkeit.» Nach einem erschöpfenden 14stündigen Arbeitstag blieb sicher weder Zeit noch Lust für «geistige Betätigung». Die nichtbetriebsgebundene Zeit reichte kaum zur Befriedigung der elementarsten Bedürfnisse - Schlafen und Essen. Von einem 
Familienleben außerhalb des Betriebes konnte kaum die Rede sein ${ }^{24}$. Trotz zunehmender Verbesserung hinsichtlich der Arbeitshygiene darf man wohl das kärgliche Leben der Weber als Zeugnis der industriellen Armut betrachten ${ }^{25}$.

Die Zustände, die für die Weber zutrafen, mögen für die Fabrikarbeiter allgemein gegolten haben, denn auch sie sind vielen Leiden ausgesetzt; besonders sind es «Rauch, Dämpfe, giftige Ausdünstungen, Mangel an Licht, Gefahr durch Maschinen verstümmelt zu werden, und die oft übermäßige Arbeitszeit» (Zuger Kalender 1869). Den Ärzten Schuler und Sonderegger waren die durch Arbeitsprozesse bedingten Schädigungen bewußt. Sonderegger war Mitglied der Kommission zur Beratung eines Gesetzesvorschlages zum Schutz der Fabrikarbeiter ${ }^{26}$. Er forderte, daß nicht mehr als elf Stunden Arbeit pro Tag verlangt werden dürften; ferner sollte eine bestimmte Freizeitregelung gewährleistet sein. Die Arbeitsräume hatten hell, trocken und gut ventiliert zu sein. Die Anfänge der Arbeiterschutzgesetzgebung in der Schweiz gehen in die dreißiger und vierziger Jahre des 19. Jahrhunderts zurück ${ }^{27}$. Nicht nur Wissenschaftler setzten sich für eine bessere Gesundheit der arbeitenden Bevölkerung ein, sondern auch Pfarrer, die sich speziell an einem Verbot der Kinderarbeit, aber auch an Maßnahmen für eine aktive Arbeitshygiene beteiligten ${ }^{28}$.

Der Beitrag der Kalender zur Gesundheitsförderung der Fabrikarbeiter bestand größtenteils aus Warnungen vor dem zu langen Aufenthalt in Fabriken, «wo zu viele Leute zusammen sind», wie der «Schaffhauser Bote» von 1872 beklagt, und «wo die Luft mit schädlichen Dünsten, Farbstoffen, Staub usw. angefüllt ist». Zur Verbesserung der mißlichen Zustände appellierte man an die verantwortliche Führung, an die «Fabrikherren», deren «heilige Pflicht» es sei, soviel wie möglich für gute Lüftung der Lokale zu sorgen und zu sehen, daß die Leute nicht über 12 Stunden arbeiten müssen. Den Fabrikarbeitern selber empfiehlt der Kalendermacher «Abwechslung im Gebrauch der Glieder und Sinne, bisweilen Arbeit im Freien, frische Luft in den Stuben und Schlafkammern, kräftige Nahrung, Reinhaltung des Körpers, ungeschmälerte Ruhe.» Solche Hinweise sollten dazu beitragen, die schädlichen Einflüsse im Fabrikleben zu mildern, auch wenn sie diese nicht zu verhindern vermochten.

Obwohl der Bergbau in der Schweiz nur aus einigen Versuchen bestand, widmet ein Kalender den Bergleuten seine Aufmerksamkeit: Im «Zuger Kalender» von 1869 heißt es, daß diese am meisten von allen Berufstätigen das Mitleid aller verdienen, da sie «innerhalb der Erdrinde ihr Leben fristen 
und bis zur wandelnden Leiche dahinschwinden». In der Tat gibt es Schädigungen des Organismus bei diesen Arbeitern in besonders großer Zahl. Im wesentlichen handelt es sich um Erkrankungen der Lunge und der Luftwege, hervorgerufen durch die Aufnahme von Gestein und Kohlenstaub und Vergiftungen; des weiteren um Hautleiden, Rheuma, Erkrankungen der Muskeln, Knochen und Gelenke ${ }^{29}$. Schon 1728 hatte Johann Friedrich Henckel in seinem Werk «Medicinischer Uffstand und Schmeltz-Bogen, insonderheit von der Bergsucht und Hütten-Katze» nachzuweisen versucht, daß die Menge der Staubpartikel von der Zahl der Arbeitsstunden abhängt ${ }^{30}$.

Daß gewerbliche Erkrankungen am deutlichsten bei den Arbeitern vorkamen, die giftige Stoffe zu gewinnen oder zu verarbeiten hatten und dabei gesundheitsschädigende Luft einatmen mußten, gab Anlaß zu häufigen Studien. Schon während des 18. Jahrhunderts befaßten sich Ärzte mehrfach mit den Krankheiten der Berg- und Hüttenarbeiter ${ }^{31}$. Für die Wiederherstellung der Gesundheit in den verschiedenen Gewerbszweigen sei besonders auf die Gründung von Krankenkassen hingewiesen. Die ersten entstanden schon im 13. und 14. Jahrhundert als sogenannte Knappschaftskassen ${ }^{32}$.

Wenn auch die Kalenderschreiber das Phänomen verschiedener Gewerbekrankheiten nicht zu beseitigen vermochten, so hofften sie doch, mit aufklärenden Artikeln wenigstens die Aufmerksamkeit der Leser zu gewinnen. Rückblickend kann als häufigste Berufskrankheit die durch Verunreinigung der Luft herbeigeführte Tuberkulose genannt werden. Nicht selten waren Erkrankungen des Magens wegen einseitiger oder schlechter Ernährung und durch Überforderung entstandene Krankheiten der Nerven.

Folgen wir den Kalendern, so war ein Teil dieser Krankheiten selbst verschuldet, denn Arbeit ohne entsprechenden Ausgleich oder ohne genügende Nahrung führt bei jedem Beruf zur Krankheit. Die gutgemeinten Ratschläge der Kalendermacher verdecken jedoch das Grundübel jener Zeit, nämlich das soziale Elend. Gerade auf diese eigentliche Ursache weisen die Kalenderschreiber nicht hin. In den Kalendern tritt deutlich die Ansicht hervor, daß ein vernünftig lebender Mensch kaum oder überhaupt nicht krank zu werden brauche. Der Boden für die Krankheit werde durch unzweckmäßige Ernährung vorbereitet. Nach ihnen zu urteilen, würde eine energische Korrektur des eigenen Lebenswandels vielen Krankheiten vorbeugen. 


\section{Anmerkungen}

${ }^{1}$ Erich Auer, Entwicklung und Stand der sozialmedizinischen Reform, Basel 1955, S.43: «Hippokrates (460-375 v.Chr.) ... äußerte sich dahingehend, daß für ihn die Berufe Krankheitsquellen im wahren Sinne des Wortes bedeuten.»

${ }^{2}$ Ulrich Ellenbog (1440-1499) studierte die Vergiftung bei Goldminenarbeitern; Leonardo da Vinci (1452-1519) beschäftigte sich mit der Bewegung des Arbeiters bei bestimmten Verrichtungen. Ebenda, S. $43 \mathrm{f}$.

${ }^{3}$ H. Buess und F. Koelsch, Geschichte der Erforschung der Berufskrankheiten, aus: Handbuch der gesamten Arbeitsmedizin, Bd. II/1, Berlin/München/Wien 1961, S.21. Ramazzini unterscheidet «zwei große Gruppen, nämlich die durch das Material (schädliche Dämpfe, kleinste Partikel) und die durch die Art der Arbeit (ungesunde Körperhaltung, heftige Bewegung usw.) bedingten Gesundheitsstörungen».

${ }^{4}$ Alfons Fischer, Geschichte des deutschen Gesundheitswesens, Bd.2, Berlin 1933, S. 253.

${ }^{5}$ E.Auer, Entwicklung und Stand..., S.44 f.; 1834 wiesen der Genfer Hygieniker HenriClermond Lombard (1803-1895) und der Berner Professor Daniel Jonquière (1821-1899) auf die durch bestimmte Berufe verursachte Lugenschwindsucht hin.

${ }^{6}$ Das eidgenössische Fabrikgesetz entstand 1877.

${ }^{7}$ J. L.Sonderegger, Vorposten der Gesundheitspflege, St. Gallen 1890, S. 364.

${ }^{8}$ F.Schuler, Ueber die Ernährung der Fabrikbevölkerung und ihre Mängel, Zürich 1882, S. 3.

${ }^{9}$ Ernst W.Baader, Gewerbekrankheiten, Berlin 1934, S. 143: «Nunmehr wissen wir, daß eine große Reihe gewerblicher Staubarten verschiedener Herkunft durch Einatmung Schädigung der Atmungswege veranlassen kann.» Im Inspektionsbezirk München wurden neben anderen Berufen auch bei Schneidern allein 154 Krankheiten auf Staubeinatmen zurückgeführt; siehe Ernst Brezina, Internationale Uebersicht über Gewerbekrankheiten, Wien 1912, S. 90 .

${ }^{10}$ C. Reclam, Lebensregeln, Berlin 1893, S. 368.

${ }^{11}$ Gottfried Braune, Topographiae medicae urbis Lipsiae specimen, Leipzig 1798, S.10; vgl. Alfons Fischer, Geschichte des deutschen Gesundheitswesens, Bd.2, S. 257.

12 J. L. Sonderegger, Vorposten, S. 372.

${ }^{13}$ Gelehrte wie der Professor der Medizin und Polizeiarzt Carl Reclam waren wohl Quelle solcher Spekulationen. Er vertrat die Ansicht, daß Gewerbetreibende mit vorzugsweise sitzender Stellung «ein grämliches Wesen und die Neigung zum Mystizismus und zur heuchlerischen Frömmigkeit» auszeichne. C. Reclam, Lebensregeln, Berlin 1877, S. 369.

14 J.L. Sonderegger, Vorposten, S. 371.

${ }^{15}$ Wohl wegen der Staublunge, was laut Statistiken ihre Lebenserwartungen erheblich verkürzt.

${ }^{16}$ Ernst Brezina, Internationale Uebersicht über Gewerbekrankheiten, Wien 1912, S.90.

${ }^{17}$ Sonderegger, Vorposten, S. 372.

18 Ebenda.

19 Ebenda.

${ }^{20}$ C. Reclam, Lebensregeln, Berlin 1877, S. 364.

${ }^{21}$ F. Schuler, Die glarnerische Baumwollindustrie und ihr Einflu $\beta$ auf die Gesundheit der Arbeiter. Mittheilungen aus den Fabrikinspectionsberichten der Jahre 1867 bis 1870 z. Hd. der medicinischen Cantonalgesellschaft in Glarus, S.91. 
${ }^{22}$ Stanislawa Muffey, Erkrankungen und Todesursachen nach dem Beruf, Diss. Düsseldorf 1962, S. 73.

${ }^{23}$ Ernst Brezina, Internationale Uebersicht, S. 86.

${ }^{24}$ Rudolf Braun, Sozialer und kultureller Wandel in einem ländlichen Industriegebiet, Zürich 1965, S. 28.

${ }^{25}$ Vgl. Walbaum, Erfahrungen auf dem Gebiete der Hygieine [!], Sanitätspolizei und gerichtlichen Medicin, Leipzig 1871, S. $33 \mathrm{ff}$.

${ }^{26}$ Paul Popp, Der Beitrag von Jakob Laurenz Sonderegger zur Sozialmedizin und Sozialpolitik, Diss. Basel 1960, S.40.

${ }^{27}$ H.Buess, Fridolin Schuler ..., ein Vorkämpfer der Sozialmedizin im 19.Jahrhundert, Sonderdruck, S. 8.

${ }^{28}$ Ebenda, S.9.

${ }^{29}$ Richard Wolf, Arbeit und Arbeitsgesellung der Zwickauer Steinkohlenarbeiter in der 2. Hälfte des 19. Jahrhunderts. In: Wissenschaftl. Zeitschr. der Humboldt-Univ. zu Berlin, Gesellschafts- und Sprachwissenschaftliche Reihe, Jg. 20 (1971), Heft 1, S. 83.

${ }^{30} \mathrm{Vgl}$. H. Buess, Geschichte der Erforschung der Berufskrankheiten, S. 22.

${ }^{31}$ So z.B. Johann Gottlieb Neumann, De praeservandis metallicolarum morbis, Halle 1721. C.L.Scheffler, Abhandlung von der Gesundheit der Bergleute, Chemnitz 1770; vgl. Alfons Fischer, Gesundheitswesen, Bd.2, S.253.

${ }^{32}$ A. Fischer, Gesundheitswesen, Bd. 1, S. 215. 
Literatur zum Thema: Arbeitshygiene in schweizerischen Kalendern des 19. Jahrhunderts

Auer, Erich, Entwicklung und Stand der sozialmedizinischen Reform in der schweizerischen Industrie. Ein Beitrag zur Geschichte der Arbeitshygiene (Basler Veröffentlichungen zur Geschichte der Medizin und der Biologie, hrsg. von H. Buess, Fasc. V), Basel 1955.

Baader, Ernst Wilhelm, Gewerbekrankheiten, Berlin 1934.

Braun, Rudolf, Sozialer und kultureller Wandel in einem ländlichen Industriegebiet im 19. und 20. Jahrhundert, Erlenbach-Zürich 1965.

Braune, Christian Gottfried Carl, Topographiae medicae urbis Lipsiae specimen, Leipzig 1798.

Brezina, Ernst, Internationale Uebersicht über Gewerbekrankheiten (Wiener Arbeiten aus dem Gebiete der sozialen Medizin, Heft 3, Nr. 33), Wien 1912.

Buess, Heinrich: Fridolin Schuler (1832-1903) aus Mollis, ein Vorkämpfer der Sozialmedizin im 19. Jahrhundert. In: Praxis, Schweizerische Rundschau für Medizin, 47.Jg., Nr.51, 18. Dezember 1958, S. 1236-1239.

Buess, Heinrich, und Koelsch, Franz, Geschichte der Erforschung der Berufskrankheiten. Sonderdruck aus: Handbuch der gesamten Arbeitsmedizin, Bd.II/1, Berlin/München/Wien 1961.

Fischer, Alfons, Geschichte des deutschen Gesundheitswesens, Bd.1 und 2, Berlin 1933.

Muffey, Stanislawa, Erkrankungen und Todesursachen nach dem Beruf, Diss. Düsseldorf 1962.

Neumann, Johann Gottlieb, De praeservandis metallicolarum morbis, Halle 1721.

Popp, Paul, Der Beitrag von Jakob Laurenz Sonderegger (1825-1896) zur Sozialmedizin und Sozialpolitik, Diss. Basel, Zürich 1960.

Reclam, Carl Heinrich, Lebensregeln, Berlin 1893.

Scheffler, Carl Lebrecht, Abhandlung von der Gesundheit der Bergleute, Chemnitz 1770.

Schuler, Fridolin, Die glarnerische Baumwollindustrie und ihr Einflu $\beta$ auf die Gesundheit der Arbeiter. Mittheilungen aus den Fabrikinspectionsberichten der Jahre 1867 bis 1870 z. Hd. der medicinischen Cantonalgesellschaft in Glarus.

Schuler, Fridolin, Ueber die Ernährung der Fabrikbevölkerung und ihre Mängel. Erstes Referat für die Jahresversammlung der Schweiz. gemeinnützigen Gesellschaft den 19. September 1882 in Glarus.

Sonderegger, Jakob Laurenz, Vorposten der Gesundheitspflege, 3. Auflage, St. Gallen 1890.

Walbaum, Carl Ferdinand Erich, Erfahrungen aus dem Gebiete der Hygieine [!], Sanitätspolizei und gerichtlichen Medicin, Leipzig 1871.

Wolf, Richard, Arbeit und Arbeitsgesellung der Zwickauer Steinkohlenarbeiter in der 2. Hälfte des 19. Jahrhunderts, in: Wissenschaftliche Zeitschrift der Humboldt-Universität zu Berlin, Gesellschafts- und Sprachwissenschaftliche Reihe, Jg. 20 (1971), Heft 1, S. 83 ff. 


\section{Summary}

The 19th century almanacs of the various regions of Switzerland were a popular way of distributing information, especially how to stay healthy and improve working conditions. Some of these almanacs even point out diseases caused by trades, which tailors, shoemakers, weavers and factory workers had to put up with daily. One example of many is the foul air that often led to pulmonary consumption. Even though the authors knew they did not have the solutions to these problems, they hoped to call the reader's attention to the fact that correction of their way of life could help prevent many illnesses.

Dr.phil. Barbara C. Hansch-Mock

Birsigstraße 127

CH-4054 Basel 